\title{
Development of Electrochemical Sensor for Simultaneous Quantification of Atenolol and Losartan Potassium
}

\author{
Swati Sharma, Nimisha Jadon*, and Rajeev Jain \\ School of Studies in Environmental Chemistry, Jiwaji University, Gwalior 474011, India
}

Received: June 11, 2018; Accepted: June 20, 2018; Published: July 31, 2018

*Corresponding author: Nimisha Jadon, PhD Assistant Professor, School of Studies in Environmental Chemistry,Jiwaji University, Gwalior-474011, Tel. No:+9107512442631,E-mail : nimisha09@yahoo.com

\begin{abstract}
Paper reports, gold nanoparticles (GNPs) /multi walled carbon nanotubes (MWCNTS) modified glassy carbon electrode (GC) sensor for the simultaneous quantification of atenolol (ATN) and losartan potassium (LOS). The modified sensor was characterized by scanning electron microscopy (SEM), electrochemical impedance spectroscopy (EIS) and cyclic voltammetry (CV). The effect of frequency, amplitude, $\mathrm{pH}$, solvent, loading of modifier and scan rate on voltammetric response of both drugs was studied to select the optimum experimental conditions. The fabricated sensor exhibited two well defined oxidation peaks at 0.9 and $1.4 \mathrm{~V}$ for ATN and LOS respectively in BR buffer $\mathrm{pH}$ 10.0. Limit of detection (LOD) for ATN $0.09 \mu \mathrm{M}$ and LOS $0.07 \mu \mathrm{M}$ and limit of quantification (LOQ) for ATN $0.26 \mu \mathrm{M}$ and LOS $0.21 \mu \mathrm{M}$ could be achieved at the fabricated sensor. ATN and LOS could be quantified in pharmaceutical formulation and spiked human serum sample at the developed sensor.
\end{abstract}

Keywords: Losartan potassium; Atenolol; GNPs/MWCNTs/GC Sensor; Voltammetry;

\section{Introduction}

Hypertension is a developing disease of therapeutic concern [1]. Atenolol (ATL) is a cardio selective $\beta$-blocker and chemically it is (RS) - 4-(2 hydroxy-3-isopropylaminopropoxy)phenylacetamide [2] (Scheme $1 \mathrm{~A}$ ). Losartan potassium (LOS) is an orally active, non-peptide angiotensin II (AII) receptor antagonist and is 2-n-butyl-4-chloro-5-hydroxymethyl-1-[2'-(1Htetrazol-5-yl) (biphenyl-4-yl) methyl] imidazole [3] (Scheme 1B).

Various analytical methods have been reported for the simultaneous quantification as well as of individual component or with other and same compounds (like ATN and LOS) such as chromatographic methods [3-19], spectroscopic techniques [2027] and electroanalytical methods [1, 28-37].Chromatographic and spectroscopic methods require, sample pretreatment, time consuming steps and high costs thus justifying the need for reliable, low cost and simpler methods. Electroanalytical

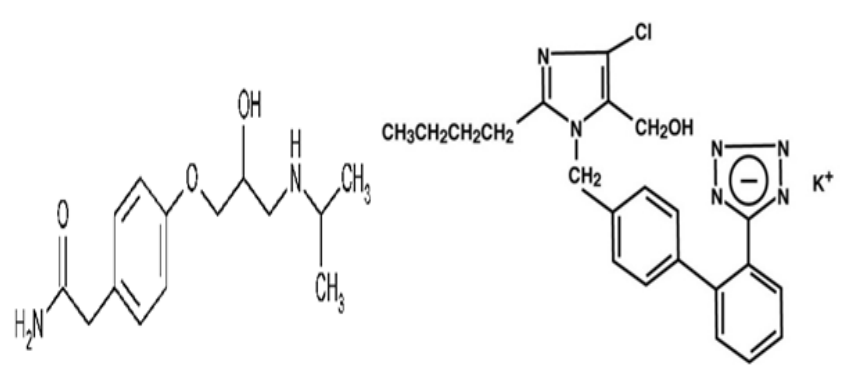

[A]

$[\mathrm{B}]$

\section{Scheme 1}

methods, being simple, economical, rapid, selective, and highly sensitive for the estimation of organic molecules including drugs in pharmaceutical formulations and human serum constitute a highly acceptable group A techniques for individual and simultaneous quantification of organic molecules [38, 45].
Gold nanoparticles (GNPs) are one of the most useful nanoparticles in industry and medicine [39, 40]. GNPs are synthesized by various methods such as [41-44]. Over the years, the unique properties of carbon nanotubes make them attractive for application as chemical sensors, in general and 
voltammetric detection, in particular [45]. GNPs have attracted much attention as a sensing platform in electrochemical sensor because of their distinctive advantages, such as easy preparation, high specific surface area, good biocompatibility and high electrical conductivity [46-48]. Modification of the sensing layer components is crucial for sensor response, for example through the incorporation of nanocomposites, the analytical performance, particularly sensitivity, selectivity, and signal to noise ratio can be significantly improved [49]. The use of (GNPs) with / (MWCNTs) based sensors have exhibited admirable electrocatalytical properties.

Analysis of compounds simultaneously, recommends lot's of benefits viz (a) less time consuming, (b) economical as more than one analyte can be quantified at the same time and (c) can also be carried out with compounds where peak-to-peak separation is very less, by modifying the experimental conditions [50].

In this paper, a simple, selective and highly sensitive voltammetric sensor based on GNPs/MWCNTs is being reported for the simultaneous determination of ATN and LOS. To the best of our knowledge, this is first systematic study of the simultaneous electrocatalytic determination of ATN and LOS in combined dosage form. The proposed method was also successfully applied to the investigation of these analytes in pharmaceutical formulation and human body fluid.

\section{Experimental Methods}

All Voltammetric measurements were carried out at ambient temperature. GNPs/MWCNTs/GC Sensor was used for the simultaneous quantification of ATN and LOS in BR buffer 10.0. The electrochemical cell contained $16 \mathrm{~mL}$ of BR buffer $\mathrm{pH} 10.0$, $2.0 \mathrm{~mL}$ acetone and $2.0 \mathrm{~mL}$ of $1.0 \mathrm{M} \mathrm{KCl}$ as supporting electrolyte.

\section{Reagents and Chemicals}

A pure standard of atenolol in powdered form was purchased from TCI Chemicals Pvt. Ltd., India and losartan potassium was obtained as a gift from Jubilant Life Sciences Ltd., Delhi, India. Analytical grade (Merck and Sigma) reagents and chemicals were used for all the experiments. All solutions were prepared in purified water (18.0 M $\Omega$ ) collected from ELGA Milli-Q system assembly. Combined dosage form of ATN and LOS manufactured from unichem laboratories Ltd India labeled as $50 \mathrm{mg}$ LOS and $50 \mathrm{mg}$ ATN, respectively, was purchased from local market. A stock solution of ATN $(7.0 \mathrm{mM})$ and LOS $(4.0 \mathrm{mM})$ was prepared in acetone and working solutions were freshly prepared daily by dilution of the stock solution.

\section{Apparatus}

All voltammetric measurements were carried out at an autolab potentiostat-galvanostat with NOVA 1.11 computrace software. Square wave voltammetry (SWV) and cyclic voltammetry (CV) were used for the investigation of these compounds simultaneously. Square wave voltammograms were recorded at a potential from 0 to $2 \mathrm{~V}$ (vs. SCE) with scan rate of $100 \mathrm{mVsec}^{-1}$, amplitude $0.04 \mathrm{~V}$ and frequency $25 \mathrm{~Hz}$. Glassy carbon electrode (GCE) behaved as working electrode, $\mathrm{Ag} / \mathrm{AgCl}$ as reference electrode and a platinum electrode as auxiliary. A calibrated Decibel DB -1011 digital pH meters fitted with a glass electrode was used for the $\mathrm{pH}$ measurements. All electrochemical measurements were performed at a room temperature $(25 \pm 0.1$ $\left.{ }^{\circ} \mathrm{C}\right)$.

\section{Synthesis of Gold nanoparticles (GNPs) and GNPs/ MWCNTs}

Sodium citrate solution $3.0 \mathrm{~mL} \mathrm{1 \%} \mathrm{(w/v)} \mathrm{was} \mathrm{added} \mathrm{to} 100$ $\mathrm{mL} 0.01 \%(\mathrm{w} / \mathrm{v})$ boiling $\mathrm{HAuCl} 4 \bullet 4 \mathrm{H} 20$ solution with magnetic stirring. Boiling continued for $8 \mathrm{~min}$. During the process, the solution colour changed from yellow to gray in $2 \mathrm{~min}$, then from gray to wine red and the mixture was further stirred for $15 \mathrm{~min}$ until the colour turned to bright red. The solution was cooled to the room temperature and kept at $4^{\circ} \mathrm{C}$. After that $2 \mathrm{mg}$ of MWCNTs was mixed in the alone prepared solution of GNPs. The solution of GNPs/MWCNTs was used as a modifier.

\section{Preparation of voltammetric sensor}

First the GCE ( $3 \mathrm{~mm}$ diameter) was polished to mirror finish with alumina powder on microfiber cloth. Next, the electrode was rinsed with purified water to clear away unbounded particles on the surface of the electrode and after that ultrasonically cleaned in ethanol and then with ultrapure water for about $5 \mathrm{~min} .6 \mu \mathrm{L}$ of the GNPs/MWCNTs solution was released on the surface of GCE and dried for $1 \mathrm{~h}$ at room temperature to form a GNPs/MWCNTs layer. Thus developed sensor was used for voltammetric investigation of ATN and LOS.

\section{Sample Preparation}

\section{Pharmaceutical Formulation}

10 tablets containing ATN and LOS were exactly weighed and finely ground with mortar pestle. A portion of powdered tablet equivalent to the average weight of one tablet was transferred into $50.0 \mathrm{~mL}$ volumetric flask using $50.0 \mathrm{~mL}$ of acetone. The mixture was sonicated for 20 min till complete dissolution. After that solution was made up to the mark using acetone to get final concentration and solution was further sonicated for $10 \mathrm{~min}$ followed by centrifugation and aliquot of the clear supernatant was transferred into an electrochemical cell for further study.

\section{Spiked human serum}

Serum sample were obtained from a local pathology clinic and stored under refrigeration. To avoid coagulation, EDTA was used as an anticoagulant. Serum matrix interferences were removed by adding buffer solution in electrochemical cell. $200 \mu \mathrm{L}$ human serum was taken in a series of centrifuge tubes and drug was added in the concentration ranges of $0.448 \mu \mathrm{M}$ to $1.792 \mu \mathrm{M}$ for ATN and $0.256 \mu \mathrm{M}$ to $1.024 \mu \mathrm{M}$ for LOS. After addition of $400 \mu \mathrm{L}$ acetone (as precipitating agent), sample was vortexed for 5-10 min and then centrifuged at $3000 \mathrm{rpm}$ for 15 min. The voltammograms were recorded by adding the collected supernatant, $16.0 \mathrm{~mL}$ BR buffer (pH 10.0) and $2.0 \mathrm{~mL}$ of $1.0 \mathrm{M} \mathrm{KCl}$. 


\section{Result and Discussion}

\section{Characterization of GNPs/MWCNTs/GC Sensor}

The voltammetric behaviour of the developed sensor was studied by using $\mathrm{K} 3 \mathrm{Fe}(\mathrm{CN})_{6}$ as a redox probe in cyclic voltammetry at $100 \mathrm{mVs}^{-1}$ scan rate. Randles-Sevcik equation was used to calculate the effective surface area:

$$
\mathrm{I}=\left(2.69 \times 10^{5}\right) \operatorname{ACD}^{1 / 2} \mathrm{n}^{3 / 2} \mathrm{v}^{1 / 2}
$$

Where, the effective surface area of the electrode in $\mathrm{cm}^{2}$ represented by $A, n$ is the number of electrons taking part in charge transfer process, $\mathrm{D}$ is the diffusion coefficient of the analyte in the solution, $\mathrm{C}$ is the concentration of $\mathrm{K} 3 \mathrm{Fe}(\mathrm{CN}) 6$ solution. The values of $\mathrm{n}$ and $\mathrm{D}$ for $\mathrm{K}_{3} \mathrm{Fe}(\mathrm{CN})_{6}$ are 1 and $7.6 \times 10^{-6} \mathrm{~cm}^{2} \mathrm{~s}^{-1}$ respectively. Surface areas of the electrodes were calculated by randles-sevcik equation and the calculated surface area of GNPs/MWCNTs/GC sensor $\left(0.020 \mathrm{~cm}^{2}\right), \mathrm{GNPs} / \mathrm{GC}$ sensor $\left(0.016 \mathrm{~cm}^{2}\right)$ and GC sensor $\left(0.010 \mathrm{~cm}^{2}\right)$ (Figure 1), indicating that GNPs/MWCNTs/GCE has largest surface area.

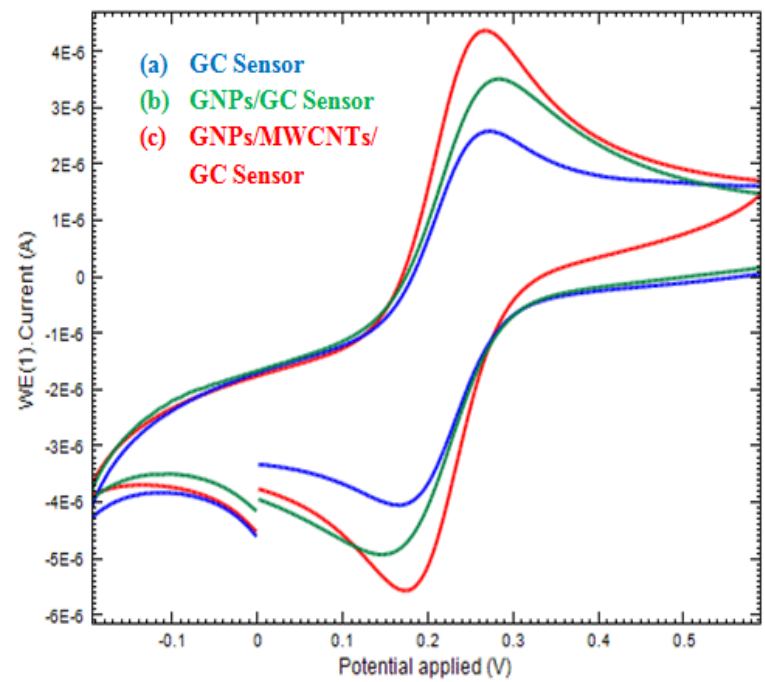

Figure 1: Cyclic voltammetric behaviour of $1.0 \mathrm{mM} \mathrm{K}_{3} \mathrm{Fe}(\mathrm{CN})_{6}$ at (a) GC Sensor (b) GNPs/GC Sensor and (c) GNPs/MWCNTs/GC Sensor

GNPs/MWCNTs/GC Sensor was also characterized by electrochemical impedance spectroscopy (EIS) (Figure 2). Nyquist plots of GNPs/MWCNTs/GC Sensor and GC Sensor in [Fe (CN)6]3 $0.1 \mathrm{M} \mathrm{KCl} \mathrm{system;} \mathrm{the} \mathrm{applied} \mathrm{DC-voltage} \mathrm{was} 0.3 \mathrm{~V}$ (vs. $\mathrm{Ag} / \mathrm{AgCl}$ ), the excitation $\mathrm{AC}$ voltage applied to the electrochemical cell was $5 \mathrm{mV}$ (peak-to-peak), and the frequency range was from $100 \mathrm{kHz}$ to $0.1 \mathrm{~Hz}$. The impedance plot consists of a semicircular part related to direct electron transfer at the GCE and [Fe

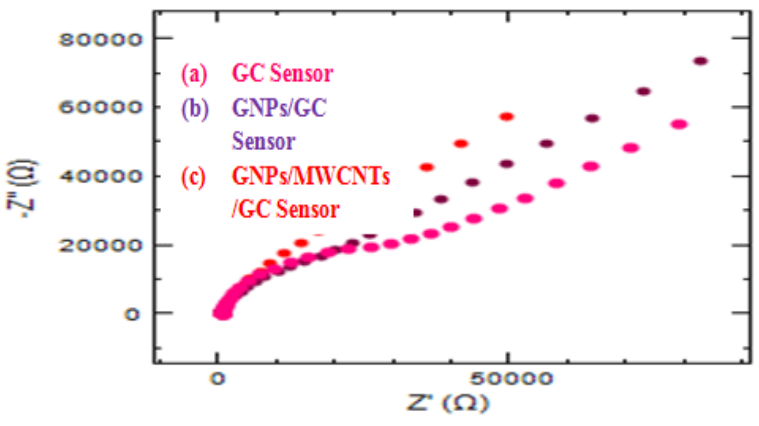

Figure 2: Nyquist plots (a) GC Sensor (b) GNPs/GC Sensor and (c) GNPs/ MWCNTs/GC Sensor in the presence of $3.0 \mathrm{mM}$ solution of K3Fe(CN)6 and $1.0 \mathrm{M} \mathrm{KCl}$

$\left.(\mathrm{CN})_{6}\right]_{3}$ and a linear part with angle $45^{\circ} \mathrm{C}$ related to diffusion control of $\left[\mathrm{Fe}(\mathrm{CN})_{6}\right]_{3}$ species at lower frequencies. Based on the charge transfer kinetics of $\left[\mathrm{Fe}(\mathrm{CN})_{6}\right]_{3}$ redox probe, the faradic impedance of unmodified and GNPs/MWCNTs/GC Sensor can be described by the Randles's equivalent circuit. On the other hand, the value of charge transfer resistance (Rct) for GCE was $39.9 \mathrm{~K} \Omega$, after modification of electrode with GNPs the value was $27.0 \mathrm{~K} \Omega$ (slightly decreased) and the value of Rct was highly decreased due to fabrication of electrode with GNPs/MWCNTs $519 \Omega$. This is confirmed by the numerical values of Rct that decreases by half of its original value of unmodified GCE. Results are consistent with $\mathrm{CV}$ data for heterogeneous charge transfer rate values. Consequently, the value of Rct for GNPs/MWCNTs/GC Sensor decreased and capacitance slightly increased. This result indicates an effective charge transfer at GCE surface via GNPs/ MWCNTs/GC Sensor mediated charge transfer mechanism.

Scanning electron microscopy (SEM) was employed to investigate the surface morphology of GNPs/GC Sensor and GNPs/MWCNTs/GC Sensor. Figure 3 shows SEM images of GNPs/GC Sensor and GNPs/MWCNTs/GC Sensor. It can be clearly seen that GNPs were highly crystalline, cube and uniformly caste on the surface of GC Sensor. The size of the GNPs varied in the range of micrometer to nanometer. MWCNTs shows a network like structure with aggregation on the surface of electrodes which indicated that, MWCNTs were immobilized on the surface of the electrode. Highly crystalline GNPs dispersed homogenously in network like structure of MWCNTs on the surface of glassy carbon electrode.

The voltammetric sensor showed a good electrocatalytic activity towards electrochemical oxidation of ATN and LOS in BR buffer pH 10. Square wave voltammetric method was carried out to investigate the oxidation behaviour of the ATN $(0.896 \mu \mathrm{M})$ and LOS $(0.512 \mu \mathrm{M})$. Maximum current was observed at the GNPs/MWCNTs/GC Sensor (Figure 4). 


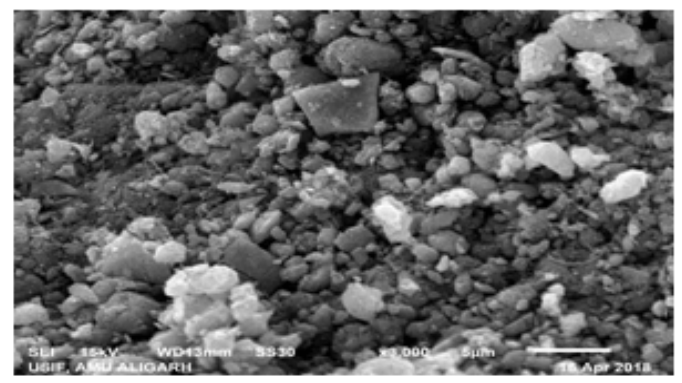

(a)

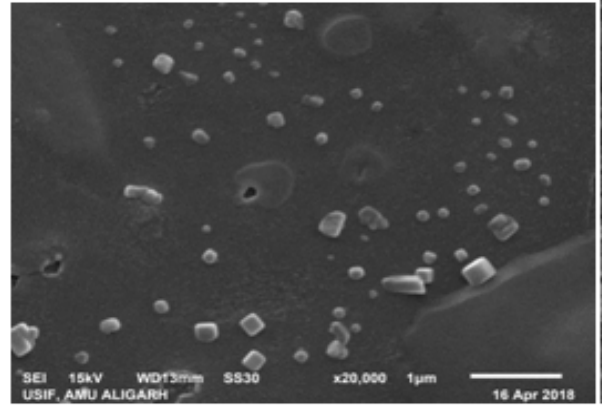

(b)

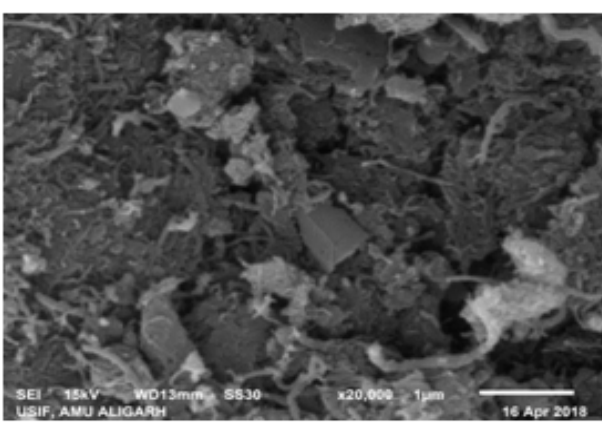

(c)

Figure 3: SEM images of fabricated GC Sensor (a) MWCNTs (b) GNPs/GC Sensor (c) GNPs/MWCNTs/GC Sensor

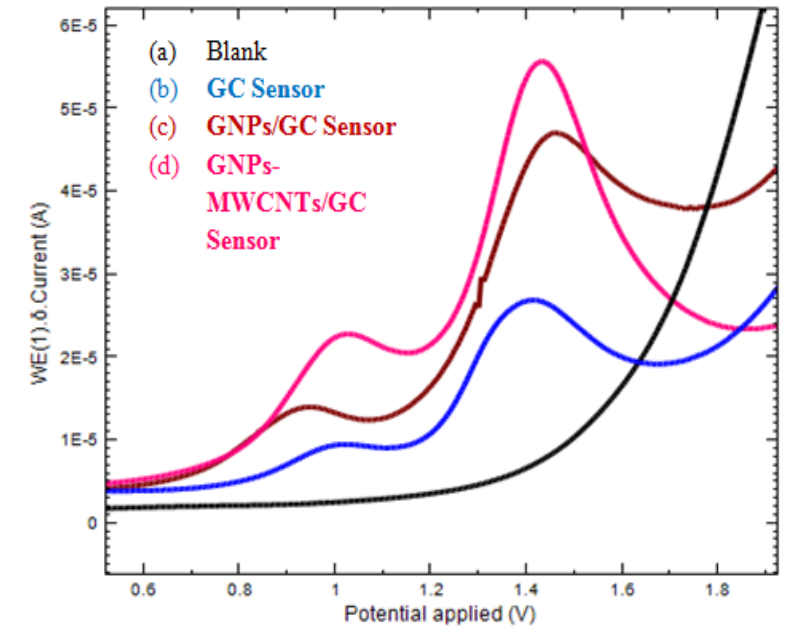

Figure 4: Square wave voltammograms of $0.896 \mu \mathrm{M}$ ATN and $0.512 \mu \mathrm{M}$ LOS in BR buffer (10.0) (a) Blank (b) GC Sensor (c) GNPs/GC Sensor and (d) GNPs-MWCNTs/GC Sensor

\section{Optimization of experimental variables}

Electrochemical investigation of these compounds was undertaken in various buffer systems such as BR and phosphate buffers maximum enhancement in the value of peak current was observed in BR buffer in the pH range from 7 to 11 at GNPs/
MWCNTs/GC Sensor. The electrocatalytic oxidation of ATN and LOS over a $\mathrm{pH}$ range (7 to 11) at developed sensor using square wave voltammetry (SWV). Figure 5 A exhibited maximum current for ATN and LOS at pH 10.0 in BR buffer. Anodic peak potential of both the analytes shifted towards less positive side with increase in $\mathrm{pH}$. The shifting of potential values clearly indicates involvement of proton in electrode process. The peak potential (Ep) Vs pH (Figure 5 B) was linear for both the oxidation peaks of ATN and LOS.

The effect of variation in frequency was studied for 1.12 $\mu \mathrm{M}$ ATN and $0.64 \mu \mathrm{M}$ LOS in the range from 5 to $35 \mathrm{~Hz}$. Figure 6 A shows that, $25 \mathrm{~Hz}$ has a maximum peak current and without any distortion sharp peak was obtained. The effect of amplitude was also examined in the range of 0.01 to $0.05 \mathrm{~V}$ (Figure 6B).

Effect of different solvents on anodic peak current of ATN and LOS at fabricated sensor was studied at highest peak current was obtained in acetone (Figure 7).

Square wave voltammetry was applied for studying the effect of loading of GNPs and GNPs/MWCNTs on the surface of the electrode. Different concentrations of GNPs $(2 \mu \mathrm{L}$ to $10 \mu \mathrm{L})$ and GNPs/MWCNTs $(2 \mu \mathrm{L}$ to $10 \mu \mathrm{L})$ of modifier was used for the electro catalytical simultaneous quantification of $1.12 \mu \mathrm{M}$ ATN and $0.064 \mu \mathrm{M}$ LOS in 10.0 BR buffer. At 10 $\mu \mathrm{L}$ concentrations of the GNPs maximum peak current could 


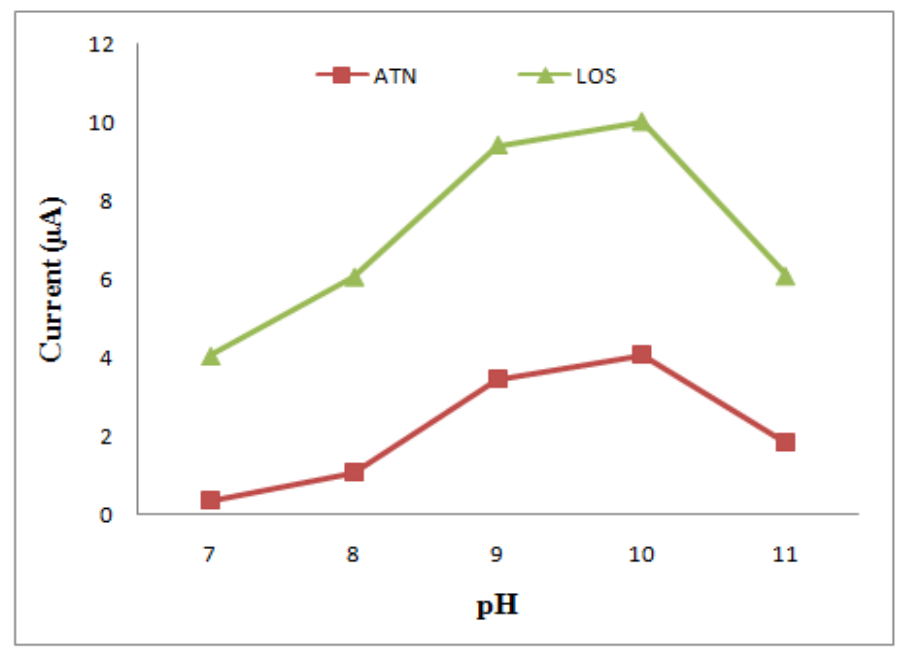

(A)

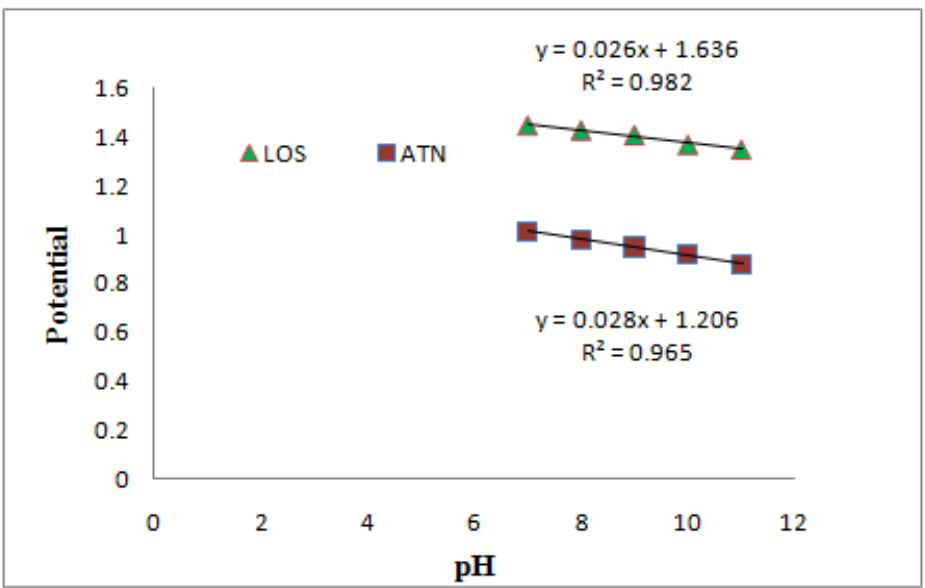

(B)

Figure 5:Effect of pH on (A) peak current and (B) potential at GNPs/MWCNTs/GC Sensor in BR buffer for ATN and LOS 


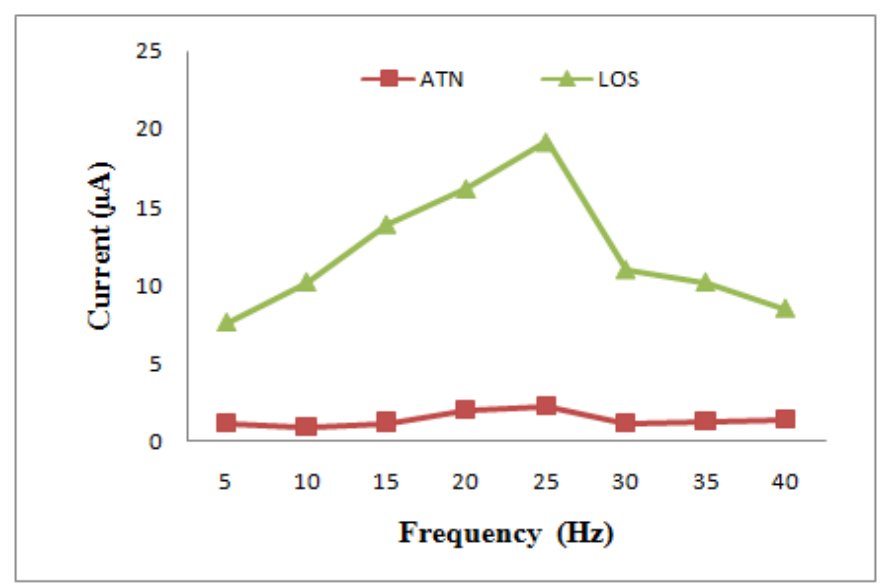

(A)

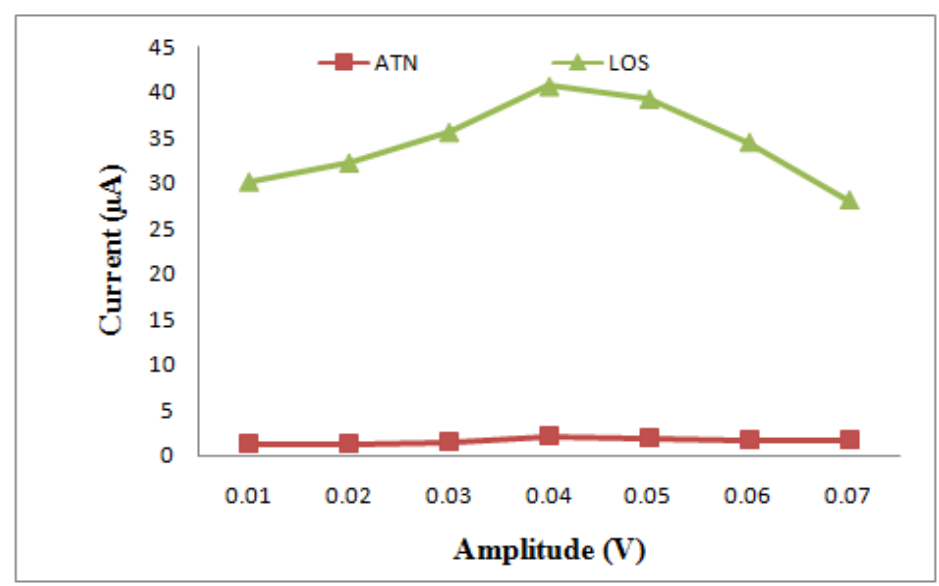

(B)

Figure 6:(A) Effect on peak current of ATN and LOS due to variation in frequency (B) due to change in amplitude

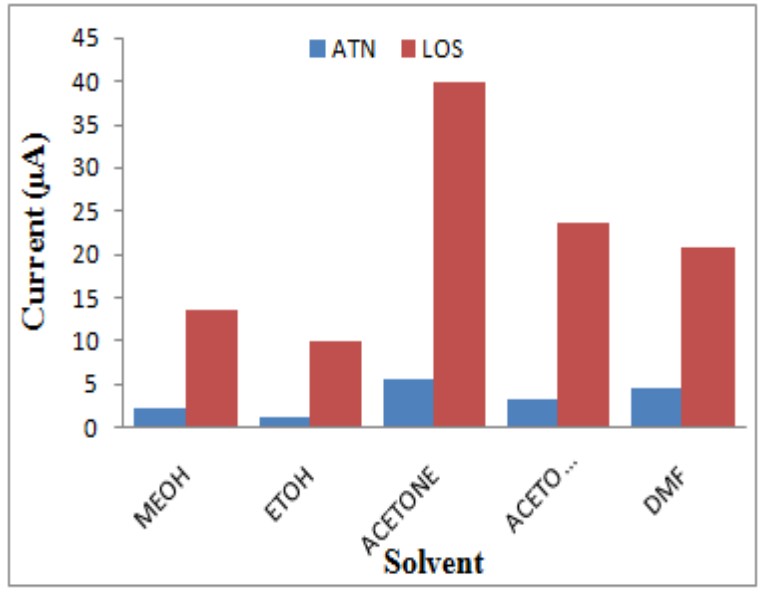

Figure 7: Effect different solvent of peak current of ATN and LOS 
be observed at GNPs/GC Sensor. Further, the nanocomposite of GNPs/MWCNTs was used for the determination of these compounds simultaneously. The maximum anodic peak current was obtained at $6 \mu \mathrm{L}$ concentration of GNPs/MWCNTs
(Figure 8). After that the examination of loading of the modifier on the surface of sensor, $6 \mu \mathrm{L}$ concentration of GNPs / MWCNTs was used for further experimental work.

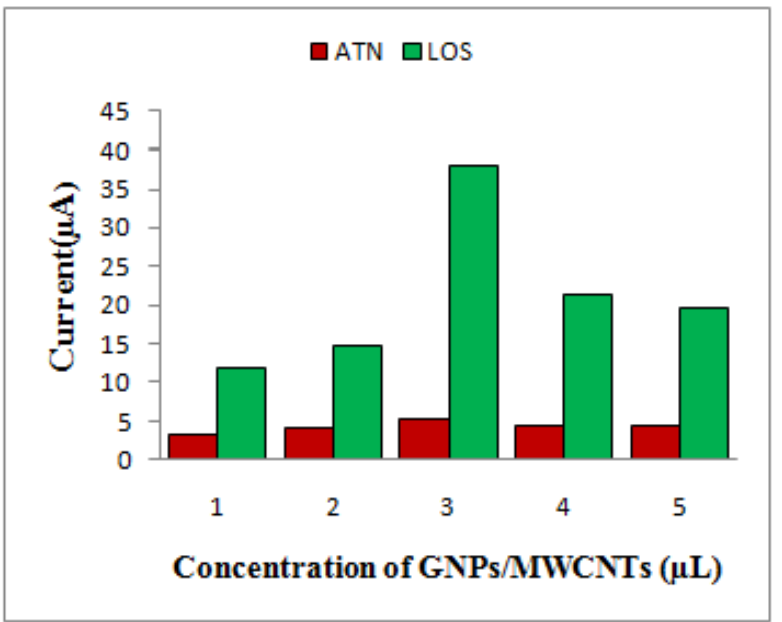

Figure 8: Effect of concentration of GNPs/MWCNTs on peak current of ATN and LOS

The dependence of scan rate on anodic peak current of ATN and LOS was optimized simultaneously at developed sensor using cyclic voltammetry (CV) in the range of 10-100 mVs-1 (Figure 9) No reduction peaks was observed in the cyclic voltammograms of ATN and LOS. The analysis of peak height against the square-root of scan rate was found to be linear indicating a diffusion controlled nature of the electrode processes (For ATN; IATN $(\mu \mathrm{A})=0.901\left(\mathrm{mVs}^{-1}\right)-1.690 ; \mathrm{r}^{2}$ $=0.997$ and $\left.\operatorname{ILOS}(\mu \mathrm{A})=2.963\left(\mathrm{mVs}^{-1}\right)-4.882 ; \mathrm{r}^{2}=0.994\right)$. The linear relationship of $\log I$ and $\log v$ can be expressed as; For ATN; $\log \mathrm{ATN}(\mu \mathrm{A})=0.5278 \log v\left(\mathrm{mVs}^{-1}\right)-1.4364$ $r^{2}=0.9909$ and for LOS; $\log \operatorname{LOS}(\mu \mathrm{A})=0.6465 \log v(\mathrm{mVs}$ $\left.{ }^{1}\right)-1.4892 ; r^{2}=0.9958$. The slope values corresponding to heterogeneous charge transfer coefficient $(\alpha)$ are very close to the theoretical value of 0.5 , which clearly indicates that the electrochemical response is controlled by diffusion mass transfer processes (Figure 10 B) [51]. Further, it indicates no significant adsorption of ATN and LOS molecules at GNPs/ MWCNTs/GC Sensor which reveal long-term stability and signal reproducibility even after several voltammetric cycles. The number of electrons transferred in the electro-oxidation processes of ATN and LOS can be estimated by applying the equation: EATNp $=0.035(\operatorname{lnv})+1.266 ; r^{2}=0.993$ and ELOSp $=0.032(\operatorname{lnv})+0.909 ; r^{2}=0.986$. The values of $\mathrm{Ep}-\mathrm{Ep} / 2$ is about 0.035 and $0.032 \mathrm{~V}$ for ATN and LOS, respectively.

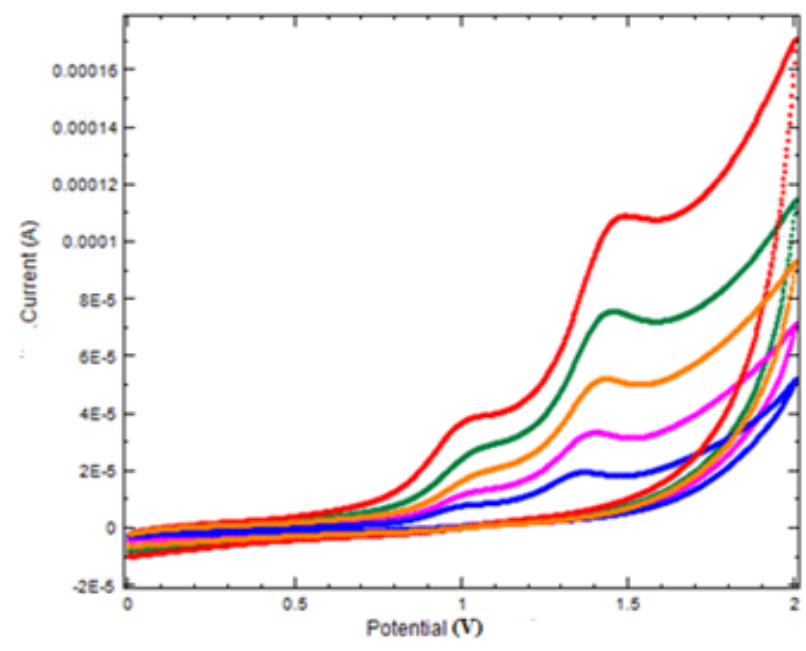

Figure 9: Effect of scan rate a) 10 b) 25 c) 50 d) 75 and e) 100mVsec-1 at GNPs/MWCNTs/GC Sensor for the oxidation of ATN and LOS 


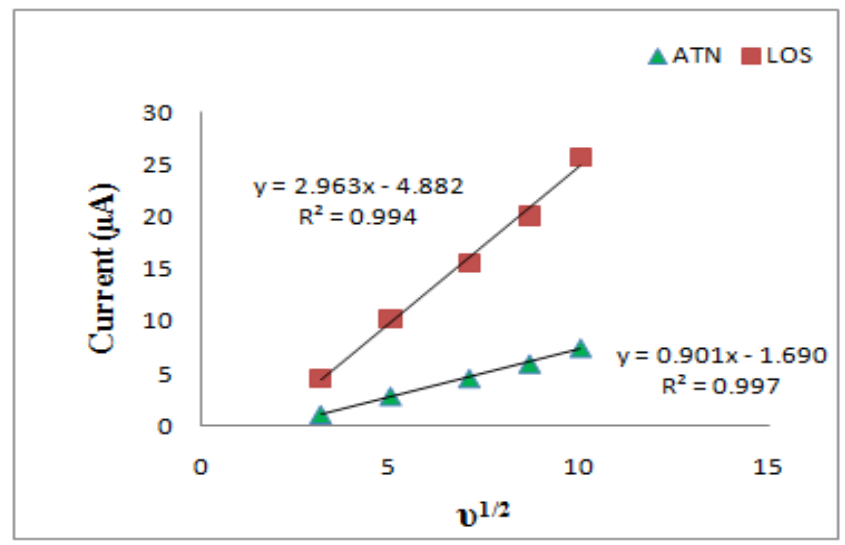

(A)

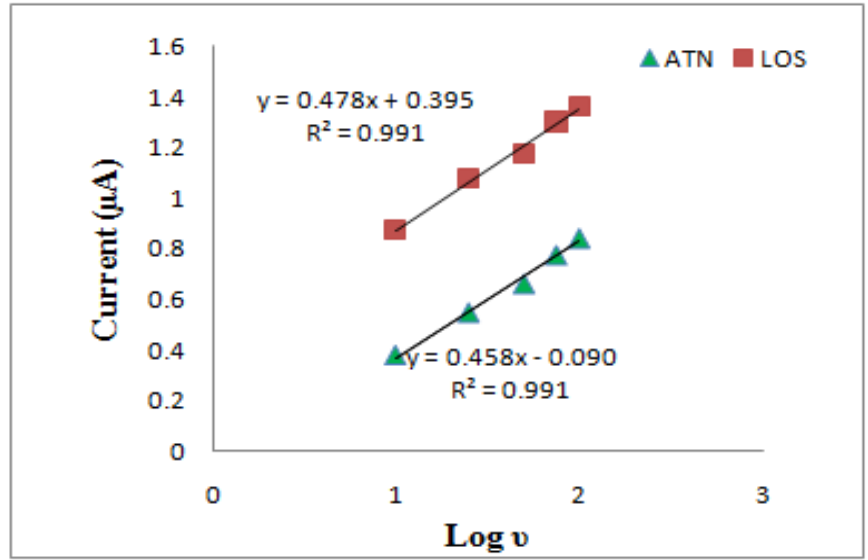

(B)

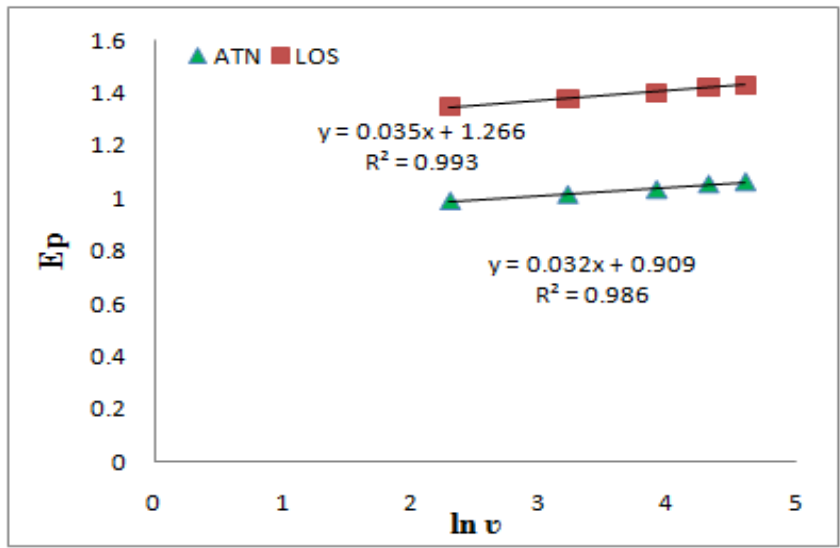

(C)

Figure 10: (A) Plot of $v^{1 / 2}$ vs ILOS and IATN (B) Plot of $\log$ I vs $\log v$ for ATN and LOS and (C) Plot of Ep vs $\ln v$ 


\section{Simultaneous determination of ATN and LOS}

Simultaneous electrochemical investigation of ATN and LOS was studied using SW voltammetric technique at GNPs/MWCNTs/GC Sensor. All the experimental parameters that affect SWV were optimized in BR buffer of pH 10.0 containing a mixture of both compounds $(7.0 \mathrm{mM}$ ATN and $4.0 \mathrm{mM} \mathrm{LOS}$ ) at modified sensor. Experimental conditions for electrochemical investigation ATN and LOS using SWV and $\mathrm{CV}$ methods have been tabulated in Table 1. ATN and LOS exhibited two well defined oxidation peaks at $0.9 \mathrm{~V}$ and 1.4 V. Various concentrations of ATN and LOS were evaluated voltammetrically at developed electrode (Figure $11 \mathrm{~A}$ ). The result showed that the plot of I vs concentration of the Analyte was linear in the range 0.112 to $1.792 \mu \mathrm{M}$ for ATN and of 0.064 to $1.0240 \mu \mathrm{M}$ for LOS respectively. The corresponding calibration equations are $\mathrm{I}_{(\mathrm{ATN})}=14.44 \mathrm{x}+6.993\left(\mathrm{R}^{2}=0.992\right)$ and $\mathrm{I}_{(\mathrm{LOS})}=24.49 \mathrm{x}+8.639\left(\mathrm{R}^{2}=0.995\right)$ respectively (Figure $11 \mathrm{~B}$ and $\mathrm{C})$.

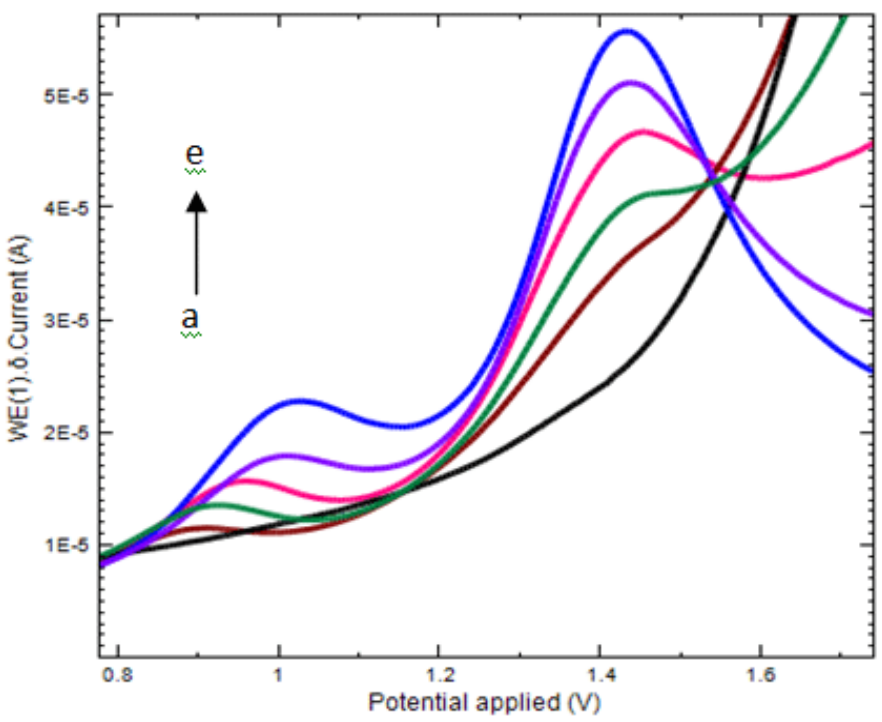

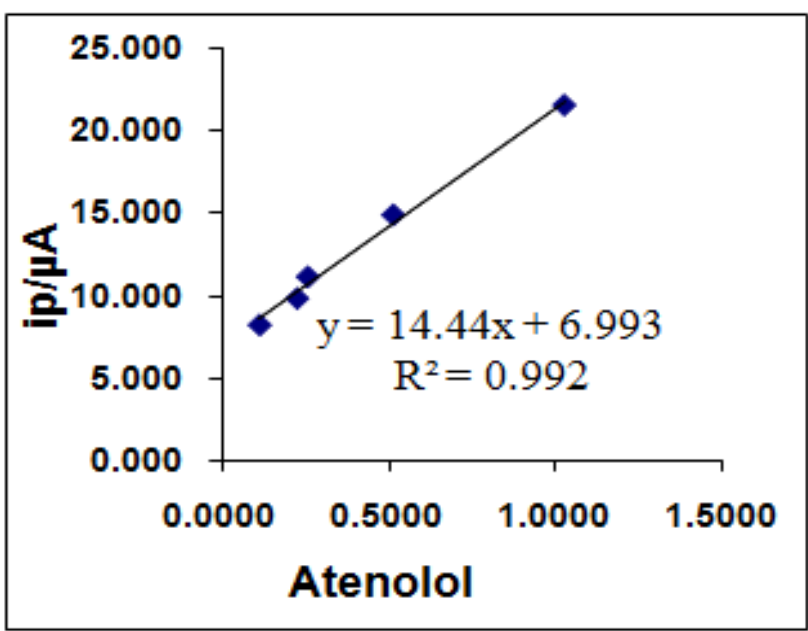

(B)

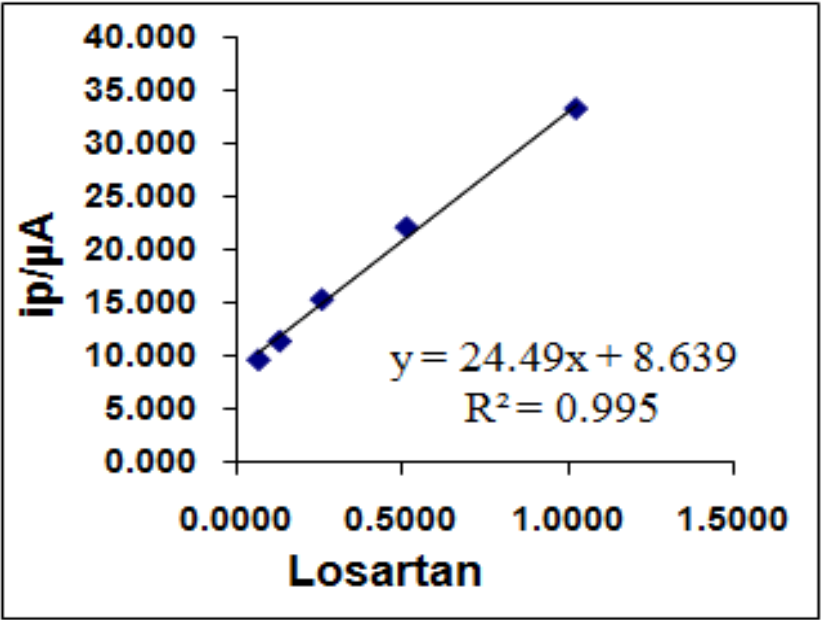

(C)

Figure 11:(A) Square wave voltammograms of ATN and LOS at GNPs/MWCNTs /GC Sensor in BR buffer (10.0) having (a to e) $0.112 \mu \mathrm{M}$ to $1.792 \mu \mathrm{M}$ for ATN and (a to e) 0.064 to $1.024 \mu \mathrm{M}$ for LOS (B) Plot of peak current as a function of concentration of ATN and (C) Plot of peak current as a function of concentration of LOS 
Table 1: Optimized experimental conditions for the determination of ATN and LOS using SWV and CV

\begin{tabular}{|c|c|}
\hline Parameters & Optimized data \\
\hline pH & 10 \\
\hline Buffer & Britton-Robinson buffer \\
\hline Solvent & Acetone \\
\hline Temperature & Room-temperature \\
\hline Frequency & $25 \mathrm{~Hz}$ \\
\hline Amplitude & $0.04 \mathrm{~V}$ \\
\hline Scan rate & $100 \mathrm{mV}$ \\
\hline Surface area of the modified & $0.020 \mathrm{~cm}$ \\
\hline sensor & 0.112 to $1.792 \mu \mathrm{M}$ \\
\hline Linear range of ATN & 0.064 to $1.024 \mu \mathrm{M}$ \\
\hline Linear range of LOS & 0.09 and $0.26 \mu \mathrm{M}$ \\
\hline LOD and LOQ of ATN & 0.07 and $0.21 \mu \mathrm{M}$ \\
\hline LOD and LOQ of LOS & \\
\hline
\end{tabular}

The limit of detection (LOD) was calculated by using formula $3 \mathrm{~S} / \mathrm{b}$, where $\mathrm{S}$ is a standard deviation of current and $b$ is a slope of the regression line. For ATN and LOS, the calculated LOD was $0.09 \mu \mathrm{M}$ and $0.07 \mu \mathrm{M}$. The limit of quantification (LOQ) was examined by the equation $10 \mathrm{~S} / \mathrm{b}$. The calculated value of LOQ $0.26 \mu \mathrm{M}$ for ATN and $0.21 \mu \mathrm{M}$ for LOS respectively. The developed method was further studied for the analysis of both the compounds in pharmaceutical formulation and human serum.

\section{Comparison of proposed method}

A comparison of the present work at GNPs/MWCNTs/ GC Sensor (BR buffer pH 10.0) with earlier investigation of ATN and LOS (individually \& simultaneously with other compounds) has been compared in Table 2. It is clear that the developed sensor is very sensitive for the quantification of ATN and LOS individually as well as simultaneously.

Table 2: Comparison of the proposed method with earlier electrochemical studies for the quantification of ATN and LOS (individually \& simultaneously with other compounds)

\begin{tabular}{|c|c|c|c|c|c|c|c|}
\hline Analyte & pH & Electrode & Technique & $\begin{array}{c}\text { Concentration range } \\
(\mu \mathrm{M})\end{array}$ & LOD $(\mu \mathrm{M})$ & LOQ $(\mu \mathrm{M})$ & Reference \\
\hline \multicolumn{8}{|l|}{ ATN } \\
\hline & PBS 7.2 & GNPs/ITO & DPV & 0.5 to 1.0 & 0.13 & & 1 \\
\hline & BR 10.0 & GNPs/MWCNTs & DPV, CA & 1 to 60 & 0.53 & & 0.53 \\
\hline & BR 9.5 & GNPs/GCE & LSV & & 0.39 & & 29 \\
\hline & BR 9.0 & MWCNTs/CPE & DPV & 5.0 to 210 & 0.29 & & 31 \\
\hline & BR 10.0 & n-MCPEs & DPV & 12 to 96 & 1.12 & 7.11 & 33 \\
\hline & $\begin{array}{c}\mathrm{H} 2 \mathrm{SO} 4 \\
\& \mathrm{NaNO} 3\end{array}$ & BDDE & SWV & 2.0 to 41 & 0.93 & & 35 \\
\hline & Acetate 5.0 & MOR/MCPE & CV, DPV & 0.4 to 80 & 0.1 & 0.35 & 36 \\
\hline LOS & BR 9.5 & BDDE & SWV, DPV & 3.0 to 74 & 0.92 & & 37 \\
\hline $\begin{array}{l}\text { ATN and } \\
\text { LOS }\end{array}$ & BR 10.0 & GNPs/MWCNTs/GCE & SWV & $\begin{array}{c}0.112 \text { to } 1.792 \& 0.064 \text { to } \\
1.024\end{array}$ & $0.09 \& 0.07$ & $0.21 \& 0.26$ & $\begin{array}{l}\text { Proposed } \\
\text { work }\end{array}$ \\
\hline
\end{tabular}

Abbreviations: GNPs: gold nanoparticles; MOR/MCPE: carbon paste electrode with mordenite zeolite; ITO: indium tin oxide; n-MCPEs: copper-oxide nanoparticles modified carbon paste electrode; BDDE: boron doped diamond electrode.

\section{Analysis of ATN and LOS in pharmaceutical formulations}

The developed electro analytical method was as tested to the quantification of ATN and LOS in pharmaceutical formulations and spiked human serum sample at fabricated sensor using SWV method (Table 3 and 4). A good recovery was observed when both of the compounds determined simultaneously in pharmaceutical formulations and spiked human serum sample.

\section{Repeatability and Reproducibility}

Reproducibility and repeatability studies of the fabricated sensor by using square wave voltammetric (SWV) technique for the determination of $0.896 \mu \mathrm{M}$ ATN and $0.512 \mu \mathrm{M}$ LOS in BR buffer $\mathrm{pH} 10.0$ have been complied in Table 5. It is clear from the table that a good reproducibility and repeatability could be achieved during the analysis of the compounds at GNPs/MWCNTs/GC Sensor. 
Table 3: Analysis of ATN and LOS in pharmaceutical formulations

\begin{tabular}{|c|c|c|c|}
\hline Compounds & Added concentration & Found concentration & \%Recovery \\
\hline ATN & 0.448 & 0.443 & 98 \\
\hline & 0.896 & 0.878 & 98.6 \\
\hline LOS & 1.792 & 1.8 & 100.5 \\
\hline & 0.256 & 0.258 & 100 \\
\hline & 0.512 & 0.512 & 100 \\
\hline
\end{tabular}

Table 4: Analysis of ATN and LOS in spiked human serum sample

\begin{tabular}{|c|c|c|c|}
\hline Compounds & Added concentration & Found concentration & \%Recovery \\
\hline ATN & 0.448 & 0.379 & 84.5 \\
\hline & 0.896 & 0.766 & 85.4 \\
\hline LOS & 1.792 & 1.700 & 94.9 \\
\hline & 0.256 & 0.258 & 92.0 \\
\hline & 0.512 & 0.468 & 91.4 \\
\hline
\end{tabular}

Table 5: Reproducibility and repeatability at GNPs/MWCNTs/GC Sensor for ATN and LOS

\begin{tabular}{|c|c|c|c|c|c|}
\hline & & $\begin{array}{c}\text { Reproducibility mean } \\
\text { current }\end{array}$ & RSD & $\begin{array}{c}\text { Single sensor } \\
\text { repeatability }\end{array}$ & RSD \\
\hline Sensor & Analyte & $\mathrm{I}(\mu \mathrm{A})$ & $\% \mathrm{RSD}$ & $\mathrm{I}(\mu \mathrm{A})$ & $\% \mathrm{RSD}$ \\
\hline Sensor 1 & ATN/ LOS & $3.16 / 7.47$ & $1.38 / 1.78$ & $3.16 / 7.47$ & $1.38 / 1.78$ \\
\hline Sensor 2 & ATN/ LOS & $4.34 / 16.0$ & $1.18 / 1.08$ & & \\
\hline Sensor 3 & ATN/ LOS & $4.49 / 30.5$ & $1.18 / 1.14$ & & \\
\hline
\end{tabular}

\section{Conclusion}

Present paper reports, a novel sensor GNPs/MWCNTs for the electro catalytic quantification of ATN and LOS by the application of SWV. The proposed method is convenient in use, rapid, very sensitive and less time consuming. The prepared sensor was further investigated in pharmaceutical formulations as well as in human serum sample for the simultaneous determination of ATN and LOS. This is the first report on examination of ATN and LOS simultaneously using electro analytical method with very low detection and quantification limit.

\section{Acknowledgment}

This work was supported by Jiwaji University, Gwalior by providing fellowship to Swati Sharma (Fellowship No. 323).

\section{References}

1. Rajendra NG, Vinod KG, Munetaka O, Neeta B. Differential pulse voltammetric determination of atenolol in pharmaceutical formulations and urine using nanogold modified indium tin oxide electrode. Electrochem. Commun. 2006;8:65-70.
2. Mojtaba S, Reza S, Mahdi Emami. A highly sensitive electrochemical sensor based on gold nanoparticles/multiwall carbon nanotubes modified glassy carbon electrode for selective determination of traces of atenolol. Anal. Methods. 2014;6 (17):7038-7045.

3. M. TS, B. EKS, Umesh C, S. SS, J. GM, B. SS. Electrochemical Investigations of Dopamine at Chemically Modified Losartan Carbon Paste Electrode: A Cyclic Voltammetric Study. Int. J. Electrochem. Sci. 2010;5:774 -781.

4. Maria AO, Jose ME, Victor C. Simultaneous determination of hydrochlorothiazide and losartan potassium in tablets by highperformance low-pressure chromatography using a multi-syringe burette coupled to a monolithic column. Anal. Bioanal. Chem. 2008;391(6):2349-2356. DOI 10.1007/s00216-008-2172-z

5. D. Durga Rao, N. V. Satyanarayana,S. S. SaitY. Ramakoti Reddy, K. Mukkanti. Simultaneous Determination of Losartan Potassium, Atenolol and Hydrochlorothiazide in Pharmaceuticals Preparations by Stability-Indicating UPLC. Chromatographia. 2009;70(3-4): 647651.

6. Sibel AO. Simultaneous determination of losartan potassium and 
hydrochlorothiazide from tablets and human serum by RP-HPLC. J. Liq. Chrom. \& Rel. Technol. 2001;24(15):2337-2346. DOI: 10.1007/ s00216-008-2172-z

7. Sathe S. R, Bari S. B. Simultaneous analysis of losartan potassium atenolol, and hydrochlorothiazide in bulk and in tablets by highperformance thin-layer chromatography with uv absorption densitometry. Acta Chromatogra. 2007;19:270-278.

8. Bryan S, Paul BC. The gas chromatographic determination of atenolol in biological samples. JPP. 1975;27(6): 430-433.

9. Orville HW, Edwin NA, William DM. Rapid determination of atenolol in human plasma and urine by high-pressure liquid chromatography. J. Pharm. Sci. 1978;67(7): 1033-1035.

10. Magnar E, Kerstin KK, Perolof L. Electron-capture-gas chromatographic determination of atenolol in plasma and urine, using a simplified procedure with improved selectivity. J. Chromatogr. B Biomed. Sci. Appl. 1990; 182(3): 341-347.

11. D. Radulovic , LJ. Zivanovic , G. Velimirovic , D. Stevanovic. High performance liquid chromatographic determination of atenolol in tablets. Anal. Lett. 1991;24(10):1813-1823.

12. $P$ Chiap, B Miralles Buraglia,A Ceccato,Ph Hubert,J Crommen. Automated liquid chromatographic determination of atenolol in plasma using dialysis and trace enrichment on a cation-exchange precolumn for sample handling. J. Chromatogr. B. 2000;739(1):205217.

13. Jorgen $H$, Anders G, Inger $H$. Direct injection of large volumes of plasma/serum on a new biocompatible extraction column for the determination of atenolol, propranolol and ibuprofen Mechanisms for the improvement of chromatographic performance. J. Chromatogr. A. 1998;797(1-2):251-263.

14. Yin-Gail Y, Peter R, T. FB. Atenolol determination by high-performance liquid chromatography and fluorescence detection. J. Chromatogr. A. 1979; 171(1):357-362.

15. Claudio G, Anna T, Sergio C, Giovanni Z. Simultaneous determination of atenolol and chlorthalidone in plasma by high performance liquid chromatography application to pharmacokinetic studies in man. J. Chromatogr. B. 1997; 698(1-2):187-194. DOI: 10.1016/S03784347(97)00298-3

16. P Chiap , Ph Hubert, B Boulanger, J Crommen. Validation of an automated method for the liquid chromatographic determination of atenolol in plasma: application of a new validation protocol. Anal. Chim. Acta. 1999; 391(2):227-238.

17. Buhring KU, Grabe A. Determination of the new \& blocker bisoprolol and of metoprolol, atenolol and propranolol in plasma and urine by high-performance liquid chromatography. J. Chromatogr.1986;382: 215-224.

18. R Ceresole, M A Moyano, M T Pizzorno, A I Segall. Validated reversed- phase HPLC method for the determination of atenolol in the presence of its major degradation product. J. Liq. Chromatogr. Relat. Technol. 2006;29(20):3009-3019.

19. Argekar A P, Powar S G. Simultaneous determination of atenolol and amlodipine in tablets by high-performance thin-layer chromatography. J. Pharm. Biomed. Anal. 2000;21(6):1137-1142.

20. Y K Agrawal , K Raman , S Rajput, S K Menon. Spectrophotometry determination of atenolol via hydroxamic acid formation. Anal. Lett. 1992;25(8):1503-1510.

21. Wenkui L, Yinghe L, Diane TF, Weng N. Hydrophilic interaction liquid chromatographic tandem mass spectrometric determination of atenolol in human plasma. Biomed. Chromatogr. 2005;19(5):385-393. doi: $10.1002 /$ bmc.462

22. El-Gindy A, Emara S, Mostafa A. HPLC and chemometric-assisted spectrophotometric methods for simultaneous determination of atenolol. amiloride hydrochloride and chlorthalidone. Farmaco .2005;60(3):269-278

23. M A Gotardo, R Sequinel, L Pezza, H R Pezza. Determination of atenolol in pharmaceutical formulations by diffuse reflectance spectroscopy. Ecl. Quim. 2008;33(4):7-12.

24. C Vetuschi, G Ragno. Fourth UV derivative spectrophotometry for the simultaneous assay of atenolol and chlorthalidone in pharmaceuticals. Int. J. Pharm. 1990; 65:171-181.

25. Mehdi A, Maryam K, Freshteh K, Mehdi B. Comparative study of first-derivative spectrophotometry and high- performance liquid chromatography applied to the determination of losartan potassium in tablets. Chem. Pharm. Bull. 2004;52(10):1166-1170.

26. Olga CL, Igor GL, Hugo JS, Renato FP, Development and validation of an UV derivative spectrophotometric determination of Losartan potassium in tablets. J. Pharm. Biomed. Anal. 2003;33(2):175-180.

27. Chaitali T, Jyoti D, P. YP. Simultaneous estimation and validation of losartan potassium and hydrochlorothiazide in bulk and tablet dosage form by using different spectrophotometric method. Der. Pharma. chem. 2014;6(2):24-30.

28. Stela P, Florina P, Camelia G, Elena MP, Liviu CB , Alexandru SB. Electrochemical investigation of atenolol oxidation and detection by using a multicomponent nanostructural assembly of amino acids and gold nanoparticles. Chem. Phys. Lett. 2011;504(1-3):56-61.

29. R. NH, B. EKS, B. SS, S. TN. Electro-oxidation of Atenolol at a Glassy Carbon Electrode. Int. J. Electrochem. Sci. 2008;3: 302-314.

30. Asma K, Sayed MG, Saeed M, Mohsen B. Multivariate curve resolutionalternating least squares assisted by voltammetry for simultaneous determination of betaxolol and atenolol using carbon nanotubes paste electrode. Bioelectrochemistry.2013;94:100-107.

31. M.Murugananthan, S.S.Latha, G.Bhaskar Raju, S.Yoshihar. Role of electrolyte on anodic mineralization of atenolol at boron doped 
diamond and Pt electrodes. Sep. Purif. Methods. 2011;79:56-62.

32. Nasrin S, Mohammad H, Lotfali S, Robab M, Abolghasem J. Electrochemical behavior of atenolol, carvedilol and propranolol on copper-oxide nanoparticles. Electrochim. Acta. 2011;58:336-347.

33. Mohsen B, Sayed MG, Ebrahim H. A Gold nanoparticle modified carbon paste electrode as a sensor for simultaneous determination of acetaminophen and atenolol. Int. J. Electrochem. Sci. 2010;5:1922 1933.

34. Elen RS, Roberta AM, Romeu CRF, Orlando FF. Square-wave voltammetric determination of propranolol and atenolol in pharmaceuticals using a boron-doped diamond electrode, Talanta. 2010;81(4-5):1418-1424.

35. Majid A, Maryam V, Maryam V. Electrochemical study of atenolol at a carbon paste electrode modified with mordenite type zeolite. Mater. Sci. Eng. C 2010;30(5):709-714.

36. Michely CGS, Cesar RTT, Luiz HDA, Elen RS. Evaluation of boron-doped diamond electrode for simultaneous voltammetric determination of hydrochlorothiazide and losartan in pharmaceutical formulations. Sens. Actuators B. 2013;188:263-270.

37. S. Azhar Ali, Atya Hassan. Cyclic voltammetric study of losartan potassium. Int. Res. J. Pure Appl. Chem. 2014;4(1): 128-136. doi: 10.9734/IRJPAC/2014/5471

38. Nimisha J, Rajeev J, Annu P, Electrochemical analysis of amlodipine in some pharmaceutical formulations and biological fluid using disposable pencil graphite electrode. J. Electroanal. Chem. 2017;788:713.

39. Imre D. Titanium dioxide and gold nanoparticle for environmental and biological application. Annals of Faculty Engineering Hunedoara 2011;1:161-166

40. Sunita R B, Veera R G, Tushar K G, Robert V T, Sudarshan K L. Gold, silver, and palladium nanoparticle/nano-agglomerate generation, collection, and characterization. J. Nanoparticle Res. 2011;13(12):6591-6601.

41. Madu AN, Njoku PC, Iwuoha GN, Agbasi UM. Synthesis and characterization of gold nanoparticles using 1-alkyl, 3-methyl imidazolium based ionic liquids. IJPS.2011; 6(4):635-640. doi: $10.5897 /$ IJPS10.487
42. O. Ya. Uryupina,V. V. Vysotskii,V. V. Matveev,A. V. Gusel'nikova,V. I. Roldughin. Production of gold nanoparticles in aqueous solutions of cellulose derivatives. Colloid J.2011;73(4):551-556.

43. N.Haustrup, G.M.O'Connor. Nanoparticle generation during laser ablation and laser-induced liquefaction. Physics Procedia. 2011;12:4653.

44. Haiss W, Thanh NT, Aveyard J, Fernig DG. Determination of size and con-centration of gold nanoparticles from UV-vis spectra. Anal. Chem.2007;79(11):4215-4221. doi: 10.1021/ac0702084

45. Nimisha J, Rajeev J, Swati S, Kshiti S. Recent trends in electrochemical sensors for multianalyte detection - A review. Talanta.2016;161:894-916.

46. Fernández F, Sánchez-Baeza F, Marco MP. Nanogold probe enhanced sur-face plasmon resonance immunosensor for improved detection of antibioticresidues. Biosens. Bioelectron.2012;34(1):151-158. doi:10.1016/j.bios.2012.01.036

47. Vigneshwaran Mani, Bhaskara V. Chikkaveeraiah, Vyomesh Patel, J. Silvio Gutkind, James F. Rusling. Ultrasensi-tive immunosensor for cancer biomarker proteins using gold nanoparticlefilm electrodes and multienzyme-particle amplification. Anal. Chem.2009;3(3):585-594. doi:10.1021/nn800863w

48. C. N. R. Rao, B. C. Satishkumar, A. Govindaraj, Manashi Nath. Nanotubes. Chem. Phys. Chem. 2001;2(2):79-105.

49. Hasan B, Niyoosha P, Bardia J, Salimeh A, Ali H, Hosein K. A novel electrochemical platform for sensitive and simultaneous determination of dopamine, uric acid and ascorbic acid based on Fe304 $\backslash \mathrm{SnO} \backslash \mathrm{Gr}$ ternary nanocomposite. Microchem J.2017;131:120-129.

50. Xia L, Wen-Jin L, Lei L, Yang Y, Lu-Gang M, Zhang P.A label-free electrochemical immunosensor based on gold nanoparticles for direct detection of atrazine. Sens. Actuators B.2014;91:408-418.

51. E.Laviron. General expression of the linear potential sweep voltammogram in the case of diffusionless electrochemical systems. J. Electroanal.chem.1979;101(1):19-28. 